\title{
A Rare Cause of Acute Abdomen: Coexistence of Acute Crohn's Disease and Gastric Perforation
}

\author{
Varlik Erol $^{\mathrm{a}, \mathrm{b}}$, Levent Ugurlu ${ }^{\mathrm{a}}$, Mutlu Unver ${ }^{\mathrm{a}}$, Safak Ozturk ${ }^{\mathrm{a}}$, Osman Bozbiyik ${ }^{\mathrm{a}}$, Semra Salimoglu ${ }^{\mathrm{a}}$, \\ Gokhan Akbulut ${ }^{\mathrm{a}}$, Cengiz Aydin ${ }^{\mathrm{a}}$
}

\begin{abstract}
Crohn's disease (CD) is an inflammatory bowel disease with the characteristics of idiopathic, granulomatous inflammation which affects any area along the gastrointestinal system from the anus to the oral cavity. We present here an unusual case of a 25 -year-old male patient, who consulted us because of abdominal pain for 2 days. The computer tomography showed free fluid at the pelvis, acute appendicitis findings and mural thickening of the terminal ileum, secondary to the appendicitis, were reported, and during the examination together with the acute abdomen findings it was decided to perform surgery on the patient. During surgery free fluid extensive inside the abdomen, at the terminal ileum indigenous for the inflammatory appearance of CD and a perforation of approximately $0.5 \mathrm{~cm}$ at the front face of the gastric pylorus, were seen. After the perforation at the stomach was primarily sutured and the inner abdomen was irrigated with physiological saline solution, so the surgery was brought to an end. After postsurgical antibiotherapy and hydration support, the patient was discharged and improved on the seventh day after surgery. Although $\mathrm{CD}$ is a chronic inflammatory disease of the gastrointestinal system, it is able to imitate the inner abdominal pathologies during an acute attack (acute appendicitis, hollow organ perforation, etc.). On patients with acute abdomen findings, where the CD diagnosis is not known, a laparotomy must be performed when a differential diagnosis of the inner abdominal pathologies cannot be made radiologically. As it is in this case, even if ileitis due to CD is determined during laparatomy, all inner abdominal organs must be explored carefully, and it should be kept in mind that the other organ pathologies can occur together. In this case the aim was to analyze the association of gastric perforation and acute $\mathrm{CD}$ at the terminal ileum of a patient with acute abdomen, where the diagnosis of $\mathrm{CD}$ is not known.
\end{abstract}

Keywords: Acute Crohn's disease; Stomach perforation; Treatment

Manuscript accepted for publication August 13, 2014

aDepartment of General Surgery, Tepecik Education and Research Hospital, Izmir, Turkey

${ }^{\mathrm{b} C o r r e s p o n d i n g ~ A u t h o r: ~ V a r l i k ~ E r o l, ~ D e p a r t m e n t ~ o f ~ G e n e r a l ~ S u r g e r y, ~ T e p e c i k ~}$ Education and Research Hospital, Izmir, Turkey.

Email: varlikerol@gmail.com

doi: http://dx.doi.org/10.14740/jcs239w

\section{Introduction}

The Crohn's disease (CD) is a chronic inflammatory bowel disease which can cause gastrointestinal indications like diarrhea and rectal bleeding, nausea, pain and abdominal cramps which generally occur together with fever, characterized with idiopathic granulomatous inflammation which can affect any area of the gastrointestinal system from oral cavity to anus, and the incidence and prevalence is increasing worldwide. Especially within the last 10 years, although medical treatment methods improved, $80 \%$ of CD patients need surgical treatment [1]. Although acute $\mathrm{CD}$ is able to imitate other pathologies of the inner abdomen which can cause an acute abdomen, often patients who received laparotomy with prediagnosis of acute appendicitis are diagnosed with CD. Despite improved diagnostic methods, it is not possible to diagnose $\mathrm{CD}$ precisely and there is not only one "golden standard" method for the diagnosis [2]. In this case, it was aimed to evaluate the association of stomach perforation together with acute $\mathrm{CD}$ at the terminal ileum at a patient with acute abdomen where the $\mathrm{CD}$ diagnosis is not known.

\section{Case Report}

A 25-year-old male patient consulted us because of abdominal

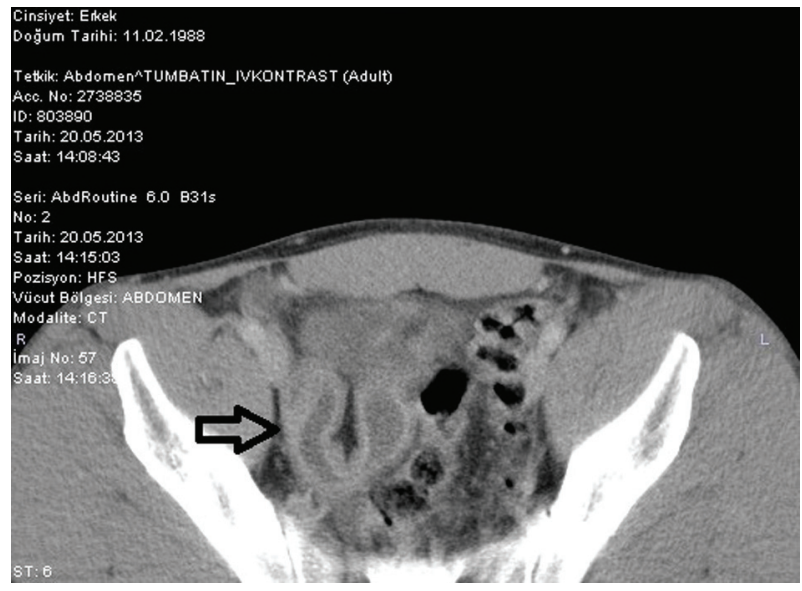

Figure 1. CT image of the edematous mural of a small bowel. 


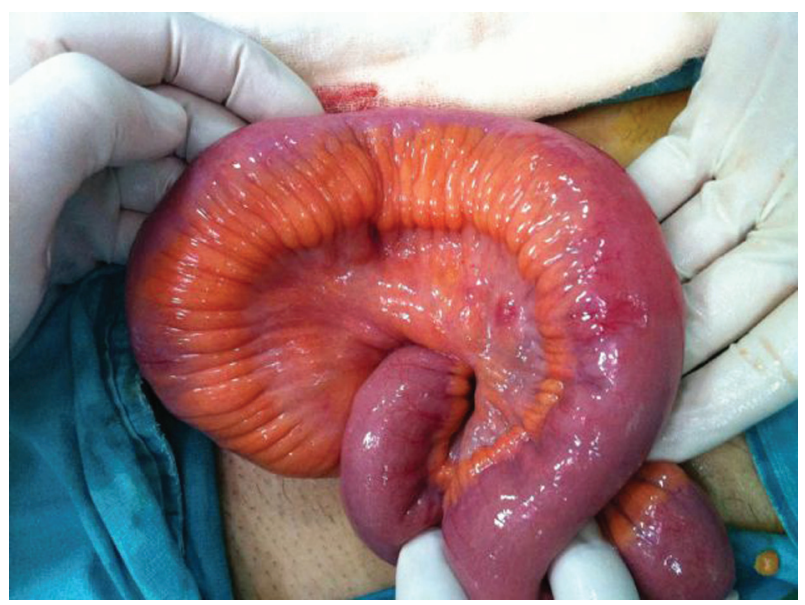

Figure 2. Intraoperative view of terminal ileum concordant with CD.

pain for 2 days. Physical examination showed extensive sensitivity of the abdomen, defence and rebound findings were present. The results of the biochemical examination were normal and at the hemogram a $15,000,000 / \mu \mathrm{L}$ white blood cell count was determined. It was reported that the ultrasound showed free fluid and indications for inflammation at the lower right quadrant of the abdomen. The computer tomography (CT) showed free fluid at the pelvis, acute appendicitis findings and mural thickening of the terminal ileum, secondary to the appendicitis, were reported (Fig. 1) and during the physical examination together with the acute abdomen findings, it was decided to perform surgery on the patient.

The operation starting with a median incision under the umbilicus, extensive inner abdominal free fluid with purulent characteristic and typical for CD inflammatory appearance at the terminal ileum was seen (Fig. 2) and on the anses of the small intestine, inflammatory and membranous tissue was determined, which is often seen at perforations of the bowel or stomach. After detecting during the exploration that the appendicitis is not inflamed, the abdominal incision was widened and the performed detailed exploration showed an approximately $0.5 \mathrm{~cm}$ perforation at the pylorus proximal of the gastric front face (Fig. 3). The gastric perforation was primarily sutured and the inner abdomen was irrigated with physiological saline solution, so surgery was brought to an end. After postsurgical antibiotherapy and hydration support, the patient was discharged on the seventh day after surgery. The biopsy taken from the patient's perioperative perforation area was reported as granulomatous inflammatory stomach tissue. The postoperative control gastroscopy showed no pathology, but as a result of the biopsy taken from the terminal ileum during colonoscopy the patient was diagnosed with $\mathrm{CD}$ and was referred to a gastroenterology clinic for maintenance treatment.

\section{Discussion}

The real cause for CD is still unknown. Genetically, microbic, immunologic, environmental, vascular and psychosocial factors, smoking, oral contraceptives, non-steroid anti-inflamma-

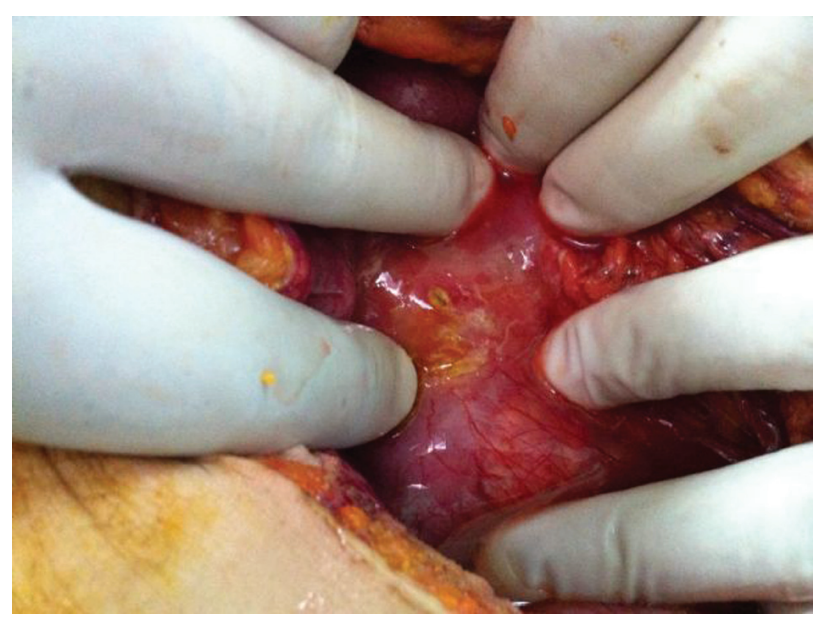

Figure 3. Intraoperative view of gastric perforation defect.

tory medications were blamed. Patients may have a tendency to an inheritance of an aberrant immunologic response caused by one or more of these provocative factors [3]. It is most frequent at the ages 15 - 30 and $60-70$ and it is bimodal. Colonic and distal CD is more seen at older people, and involvement of the ileum is more often seen at young patients [4]. Women have it 1.1 - 1.8 times more often than men [5]. The disease causes generally complaints like fever with low temperature, abdominal pain and long lasting diarrhea and weight loss. CD appears at $45 \%$ at the ileum and colon, $20 \%$ only at the colon, $33 \%$ at the small intestine and 5\% gastroduodenal area and perianal area [6]. CD's typical characteristic is remission and relapses. Complications which need surgery may occur with many patients. For patients with chronic or nocturnal diarrhea, abdominal pain, intestinal obstruction, weight loss, fever or night sweating, the diagnosis of CD should be considered [7]. Together with this, while the disease continues with attacks and remissions, sometimes it can be difficult to get a diagnosis. $\mathrm{CD}$ is a chronic inflammatory disease of the gastrointestinal system and is able to imitate other inner abdominal pathologies during an acute attack (acute appendicitis, hollow organ perforation, etc.). Episodic abdominal pain by way of cramps is the most common indication of $\mathrm{CD}$ [8]. $\mathrm{CD}$ at the terminal ileum is mostly diagnosed during the exploration which is performed with the suspicion of appendicitis and the prognosis is very good. The acute disease is generally treated conservatively and two-thirds of the patients may not show indication for regional enteritis [4]. Also during the diagnosis, diseases like appendicitis, small intestine obstruction, ulcerative colitis, irritable bowel syndrome, infectious or ischemic colitis, malignancy, hemorrhoids and diverticular diseases must be considered [8]. Biochemical examinations can be normal, also diarrhea secondary electrolyte anomalies, anemia due to vitamin B12 deficiency, high erythrocyte sedimentation rate and leukocyte may be determined. Together with present clinical findings, it is possible to make use of examinations like colonoscopy, gastroscopy, CT, barium radiography, and magnetic resonance imaging. Mural thickening of the intestine, intestinal obstruction, mesenteric oedema, abscess and fistula may be monitorized (Fig. 1). On patients with acute abdominal pain where the diagnosis of CD is not known, laparotomy must be performed in 
cases where radiologically the differential diagnosis of the inner abdominal pathologies cannot be performed. As it is in this case, even if ileitis due to CD is determined during laparotomy, inner abdominal organs should be explored carefully, and it should not be forgotten that other organ pathology can occur in conjunction. On patients with perioperative inflammatory intestinal disease findings, control colonoscopy and biopsy must absolutely be performed during the postoperative period to clear the diagnosis.

$\mathrm{CD}$ is a disease which can cause inflammation at any area of the gastrointestinal system, mostly at the ileum. The disease can show many clinical indications and it can imitate other inner abdominal pathologies. Although screening and laboratory examinations have improved, there is no golden standard method which can be used alone for the diagnosis. Even if with screening methods (CT) CD is suspected especially at patients who consult with acute CD attacks and at patients without definite diagnosis, if there is no remission at the examination findings and the laboratory findings of the patient during the follow-up, it must be kept in mind that it can occur together with other inner abdominal pathologies. When exploration is performed on a patient because of acute abdominal findings, the whole gastrointestinal system should be examined carefully for other possible pathologies. If at the intestinal segment where CD is determined there are no complications like obstruction, perforation or fistula, no interference should be made to the acute sickly segment.

\section{Conflict of Interest}

The above authors have no potential conflicts to disclose.

\section{References}

1. Larson DW, Pemberton JH. Current concepts and controversies in surgery for IBD. Gastroenterology. 2004;126(6):1611-1619.

2. Stange EF, Travis SP, Vermeire S, Beglinger C, Kupcinkas L, Geboes K, Barakauskiene A, et al. European evidence based consensus on the diagnosis and management of Crohn's disease: definitions and diagnosis. Gut. 2006;55(Suppl 1):i1-15.

3. Thoreson R, Cullen JJ. Pathophysiology of inflammatory bowel disease: an overview. Surg Clin North Am. 2007;87(3):575-585.

4. Kornbluth A, Sachar DB, Salomon P. Crohn's disease. In: Feldman M, Scharschmidt BF, Sleisenger MH, eds. Sleisenger \& Fordtran's Gastrointestinal and Liver Disease: Pathophysiology, Diagnosis, and Management. Vol 2. 6th. Philadelphia, Pa: WB Saunders Co; 1998:17081734.

5. Panes J, Gomollon F, Taxonera C, Hinojosa J, Clofent J, Nos P. Crohn's disease: a review of current treatment with a focus on biologics. Drugs. 2007;67(17):2511-2537.

6. Wilkins T, Jarvis K, Patel J. Diagnosis and management of Crohn's disease. Am Fam Physician. 2011;84(12):13651375.

7. Hanauer SB, Sandborn W. Management of Crohn's disease in adults. Am J Gastroenterol. 2001;96(3):635-643.

8. Knutson D, Greenberg G, Cronau H. Management of Crohn's disease--a practical approach. Am Fam Physician. 2003;68(4):707-714. 